\title{
Kernos
}

Revue internationale et pluridisciplinaire de religion grecque antique

$22 \mid 2009$

Varia

\section{Barbara Kowalzig, Singing for the Gods. Performances of Myth and Ritual in Archaic and Classical Greece}

\section{Claude Calame}

\section{(2) OpenEdition \\ 1 Journals}

\section{Édition électronique}

URL : http://journals.openedition.org/kernos/1795

DOI : 10.4000/kernos. 1795

ISSN : 2034-7871

\section{Éditeur}

Centre international d'étude de la religion grecque antique

\section{Édition imprimée}

Date de publication : 1 janvier 2009

Pagination : 317-320

ISSN : 0776-3824

\section{Référence électronique}

Claude Calame, «Barbara Kowalzig, Singing for the Gods. Performances of Myth and Ritual in Archaic and Classical Greece », Kernos [En ligne], 22 | 2009, mis en ligne le 15 septembre 2011, consulté le 21 septembre 2020. URL : http://journals.openedition.org/kernos/1795 ; DOI : https://doi.org/10.4000/ kernos. 1795

Ce document a été généré automatiquement le 21 septembre 2020 


\title{
Barbara Kowalzig, Singing for the Gods. Performances of Myth and Ritual in Archaic and Classical Greece
}

\author{
Claude Calame
}

\section{RÉFÉRENCE}

Barbara KowALzIG, Singing for the Gods. Performances of Myth and Ritual in Archaic and Classical Greece, Oxford, Oxford University Press, 2007. 1 vol. 14 渵 $22 \mathrm{~cm}, 528$ p. (Oxford Classical Monographs). ISBN : 978-0-19-921996-4.

1 Dans un essai récent d'«anthropologie historiographique", Marshall Sahlins s'interroge à nouveau sur les acteurs de l'histoire dans la relation dialectique entre structure et événement ${ }^{1}$. Dans une comparaison entre "the Peloponnesian war » et «the Polynesian war » (entre la guerre du Péloponnèse telle qu'elle est mise en scène par Thucydide et une analogue entreprise impérialiste conduite par un royaume insulaire dans l'archipel des Fidji au $\mathrm{XIX}^{\mathrm{e}}$ siècle), cette nouvelle enquête d'histoire anthropologique se fonde sur le concept anglo-saxon de "agency». Au-delà des contingences de l'événement, l'histoire est faite par des agents à considérer en tant qu'individus, groupes sociaux ou collectivités ethniques; dans leur action historique, ces différents acteurs sont soumis aux mêmes valeurs et déterminations d'ordre culturel : perspective anthropologique oblige !

2 Aux agents de l'histoire politique et sociale des cités grecques, l'étude de B.K. nous invite à ajouter désormais les groupes choraux et leurs poètes : en général des groupes de fils de citoyens ou de filles de citoyens chantant et dansant, aux grandes occasions cultuelles inscrites dans le calendrier de la cité, des compositions appartenant aux différents genres de la poésie rituelle qu'est le mélos. Parfois anonymes, ces poèmes 
relevant de l'art des Muses sont composés par des artisans, créateurs inspirés qui n'appartiennent pas forcément à la communauté civique à laquelle l'exécution chantée de ces compositions est destinée. Si différentes procédures énonciatives ancrent ces poèmes dans le hic et nunc de leur "performance » en faisant d'eux de véritables actes (rituels) de chant, leur composante narrative les réfèrent au passé héroïque de la communauté. Ainsi l'enquête proposée par B.K. sur «singing for the gods » est une invitation à revisiter, par des exemples concrets, la question controversée (s'il en est une en anthropologie culturelle) de la relation entre le «mythe » et le " rite »; ou plutôt la relation entre d'une part la référence narrative au temps fondateur de la communauté célébrée dans les chants choraux et d'autre part la conjoncture cultuelle et civique consacrée par l'exécution d'un chant rituel à forte dimension pragmatique. La démarche est d'autant plus pertinente que, par le biais de l'étiologie, la légitimation par la référence au passé héroïque de l'occasion rituelle présente et de sa conjoncture historique (et culturelle) est volontiers inscrite dans le poème lui-même. Mais, complète B.K., par l'action "performative " chantée il ne s'agit pas uniquement de justifier le présent, mais aussi de le transformer: le mythe agi dans le rite doit être considéré en sa qualité de facteur de changement social et politique. Le lecteur critique ajoutera quant à lui que c'est la forme poétique et musicale qui, insérant le récit dans une performance d'ordre rituel, confère à la référence narrative au passé héroïque son efficacité religieuse, sociale et institutionnelle.

C'est ainsi que, tour à tour, la documentation revisitée avec profit par B.K. nous entraîne successivement sur l'île de Délos où le chant choral sous l'égide d'Apollon donne le modèle métaphorique à l'organisation politique de la Ligue de Délos; dans la plaine d'Argos dont le territoire est balisé par les offrandes musicales aux différentes divinités qui en assurent la cohésion politique dans une sorte d'amphictionie; à Égine où le soutien du sanctuaire oraculaire de Delphes conduit à l'affirmation chorale d'une forme locale de prétention panhellénique et d'identité grecque face à Athènes; sur la grande île de Rhodes dont le sanctuaire central de Lindos promeut une forme de synécisme insulaire par poésie narrative et chorale interposée, en revisitant la tradition légendaire locale (entre héros immigré et figure autochtone); en Grande Grèce aussi et singulièrement à Métaponte par la référence rituelle et musicale à la fondation achéenne pour conférer à un groupe de cités coloniales une identité rivale de l'identité ionienne imposée par Athènes; avec un retour final sur le continent, en Béotie dont les petites cités groupées autour du Lac Kopaïs déploient différents efforts cultuels pour se fabriquer, par un réseau de rites et de mythes étiologiques, une identité commune face à l'hégémonie thébaine.

4 Remarquablement fouillée et érudite, parfois quelque peu verbeuse dans l'explicitation des effets pratiques des relations tirées entre récits légendaires locaux et cultes à portée politique, la recherche se distingue par deux traits dignes d'éloge. D'une part, on ne dira jamais assez les mérites d'une recherche en anthropologie historique de la religion politique grecque qui n'élude aucune de nos sources documentaires : poèmes chantés appartenant aux différentes formes rituelles du mélos - cela va sans dire -, mais aussi données historiographiques, rapports de fouilles et reconstructions archéologiques, documents épigraphiques et, dans une moindre mesure, représentations iconographiques; la lecture de chacun de ces indices et de chacune de ces mises en forme singulières de pratiques politico-religieuses impliquent un savoirfaire particulier, déployé dans chaque cas avec pertinence et intelligence. Par ailleurs les excellentes cartes où sont reportés, dans l'état actuel de nos connaissances, les 
différents réseaux cultuels et politiques tracés par les mythes étiologiques en performance poétique et rituelle portent la marque d'un véritable travail de terrain, comme le sont aussi les photographies des paysages cultuels, prises par l'auteure ellemême. B.K. a compris que, même si elle porte sur une culture distante dans le temps, l'anthropologie culturelle et sociale ne peut se dispenser d'une exploration des lieux de déploiement des pratiques rituelles et des manifestations symboliques qui forment ses objets traditionnels. La relation est certainement forte entre l'expérience directe des espaces cultuels arpentés de manière systématique et la position épistémologique adoptée dans une recherche d'histoire anthropologique du polythéisme grec constamment sensible aux implications concrètes, pratiques de chants rituels créés par de très grands poètes.

5 Par manque de place, on illustrera l'originalité de la démarche proposée par un seul exemple. Qui se rend de nos jours du Pirée à Égine par le service maritime public est frappé par le contraste fort entre la ville tentaculaire avec le port désormais couvert de béton qu'il vient de quitter et une bourgade à peine touchée par un tourisme resté très local. Il est donc difficile d'appréhender et de se représenter à partir du contexte historique et géographique contemporain les enjeux de la rivalité proverbiale, à l'issue des guerres médiques, entre d'une part l'Athènes qui s'est faite la championne de la défense des Grecs face au barbare et qui commence à étendre son contrôle politique et économique sur la mer Égée, et d'autre part une Égine qui, sinon dans les restes des sculptures du temple d'Aphaia, nous apparait comme bien provinciale.

6 C'est ici qu'il convient de suivre attentivement B.K. dans le cheminement qu'elle propose. Du point de vue géographique, l'itinéraire trouve son point de départ dans le sanctuaire de Zeus Hellanios et du point de vue temporel dans la naissance d'Éaque, le fils du même Zeus et de la nymphe éponyme de l'île, Aigina. Avec sa fonction étiologique, le récit héroïque mettant en scène le salut qu'Éaque, avec l'aide de son père Zeus, assure à (presque) tous les Grecs à la suite d'une longue période de sécheresse est repris non seulement dans différentes épinicies de Pindare chantant la victoire d'un athlète éginète aux jeux panhelléniques, mais aussi dans le fameux sixième Péan destiné aux Delphiens en l'honneur d'Apollon Pythien. L'exécution de ce chant de culte nous invite aux Théoxénies de Delphes et il permet d'affirmer la prétention des Éacides et des Éginètes à étendre leur influence sur cette célébration panhellénique, notamment dans la perspective d'une nouvelle définition de l'identité "grecque " à l'issue des guerres médiques. Mais pourquoi le choix d'un festival appelant de bonnes récoltes en faveur de toute la Grèce ? La tentative identitaire est non seulement parallèle à la mainmise sur Délos et les Délia en l'honneur d'Apollon par les Athéniens, soucieux d'étendre leur pouvoir dans le bassin de la mer Égée; mais elle correspond aussi à un opération d'ordre économique : au centre du Golfe de Saronique, Égine apparaît en effet dès la fin $\mathrm{du} \mathrm{VI}^{\mathrm{e}}$ siècle comme un lieu privilégié pour le commerce du blé. Ce contrôle du trafic de la denrée alimentaire de base semble exprimé par le récit du vol à Épidaure des statues en bois d'olivier attique de Damia et d'Auxésia. Quant au destin de l'Éacide Néoptolème, ce qui compte ce sont moins les versions divergentes au sujet de sa mort à Delphes (la controverse à ce propos est sans issue) que le rôle de théore qu'il assume vis-à-vis d'un sanctuaire où il reçoit fonction cultuelle et honneurs héroïques.

7 Dans ce remarquable essai d'anthropologie historique et religieuse sur le rôle joué par Égine à l'issue des guerres médiques, notamment en raison de sa position géographique face à Athènes, on remarque que, par l'intermédiaire de récits mythiques à portée 
étiologique et par celui des groupes choraux qui insèrent leur narration poétique dans le culte (en faisant de leur exécution chantée des actes rituels), ce sont aussi les dieux locaux qui deviennent les acteurs d'une histoire que ses protagonistes tentent d'orienter. À ce propos, on se demandera pourquoi Aphaia, la déesse qui domine l'île en face de Zeus Hellanios, est à peine mentionnée (p. 207 n. 70 et p. 209). Si de nos jours encore son sanctuaire impressionne et par son architecture et par ses sculptures, si de manière très énigmatique les choreutes de l'Hippolyte d'Euripide (v. 1123-30) situent à Trézène une Athéna Hellania qu'elles mettent en relation avec Dictynna, Pausanias (II, 30, 3-5) ne manque pas d'une part d'établir un rapport spatial fort entre le sanctuaire d'Aphaia et celui de Zeus Hellanios; d'autre part le Périégète fait d'Aphaia, par récit étiologique interposé, la représentante éginète (par la volonté d'Artémis) de Britomartis, la nymphe devenue la Dictynna crétoise. Il conviendrait donc de penser Zeus Hellanios et Aphaia en termes de complémentarité de compétences quant au domaine maritime et au territoire cultivé de l'île; le modèle serait ici le couple que forment Athéna et Poséidon en tant que divinités tutélaires d'Athènes, assurant dans l'affirmation étiologique de l'autochtonie des Athéniens la productivité du sol de l'Attique et la domination économique sur la mer Égée. Par ailleurs pourquoi ignorer l' Épinicie 13 de Bacchylide composée pour célébrer la victoire d'un jeune Éginète au concours du pancrace des jeux de Némée peu avant 480? Déroulant l'arbre généalogique qui des parents divins d'Éaque conduit aux deux protagonistes centraux de la guerre de Troie que sont Achille et Ajax, traversé par l'isotopie de la gloire héroïque, le poème chanté par un groupe choral de jeunes gens d'Égine s'achève sur l'évocation d'Athéna, la déesse tutélaire de l'entraîneur athénien du jeune athlète, tout en insistant sur le caractère panhellénique des jeux athlétiques. Manière de conférer aux exploits des descendants d'Éaque jusque dans le présent, par la proclamation poétique, une portée panhellénique.

8 Mais, dans cette histoire manifestement orientée par les interventions chorales dans les cultes rendus aux divinités tutélaires des cités grecques classiques, quels sont en définitive les agents déterminants? De ce point de vue, les conclusions tirées des poèmes qui ont nourri le chapitre consacré à la politique panhellénique conduite par les grandes familles de l'île d'Égine sont marquées par l'hésitation. Tour à tour, B.K. évoque les figures de poètes (Pindare, Euripide), les compositions poétiques (le péan, l'épinicie), la performance cultuelle de ces chants. Elle ne mentionne que furtivement les groupes choraux (p. 219-223), représentants de la communauté civique auxquels le poète délègue volontiers sa voix. Ce sont pourtant eux qui, par le biais de la " délégation chorale » et surtout dans et par l'exécution rituelle du chant, agissent avec l'appui des divinités dont ils invoquent le pouvoir pour en faire implicitement des acteurs de l'histoire.

Le flou qui règne parfois quant à une approche centrée sur une pragmatique hypertrophiée du rituel au détriment de ses dimensions sémantiques n'enlève rien à la précision et à la cohérence d'analyses aptes à nous engager à repenser nos manières de faire l'histoire (anthropologique) de la religion grecque. "Continental theory»? Il s'agit en fait de théorie induite par la pratique des documents et du terrain, de théorie appliquée, à l'anglo-saxonne; cette théorie mise en pratique peut lever une bonne partie des objections formulées dans le contexte du scepticisme postmoderniste quant à la pertinence et à l'utilité des concepts opératoires. Les itinéraires d'anthropologie historique auxquels nous invite la belle enquête de B.K. à travers certaines parmi les 
manifestations culturelles les plus déterminantes et les plus originales de la Grèce classique valent sans aucun doute la peine d'être suivis avec beaucoup d'attention.

\section{NOTES}

1. M. SAHLIns, Apologies to Thucydides. Understanding History as Culture and Vice Versa, Chicago \& London, The University of Chicago Press, 2004.

\section{AUTEURS}

\section{CLAUDE CALAME}

École des Hautes Études en Sciences Sociales, Paris 